\title{
Surveillance system using HL7 CDA in Korea
}

\author{
Jong-Ho Lim ${ }^{1}$, Jun-Hyun Song ${ }^{1}$, Sung-Hyun Lee ${ }^{1}$, II-Kon Kim ${ }^{1}$, Byoung-Kee Yi ${ }^{2}$, Sun-Hee Park ${ }^{3}$ \\ ${ }^{1}$ Kyungpook National University, Daegu \\ ${ }^{2}$ Samsung Medical Center, Seoul, Korea \\ ${ }^{3}$ Korea Centers For Disease Control \& Prevention, Chungbuk, Korea
}

\begin{abstract}
Objective: The current process for reporting infectious disease in Korea is a complex workflow based on manual entry and verification of data and requires transmission of report via obsolete technologies such as FAX. As such, it incurs unnecessary time and effort that hinder real time monitoring of epidemic outbreak. Further, the lack of standardized coding of data in the report makes it difficult to manage and analyze the data from different sources. We propose an interoperable infectious disease reporting system based on $\mathrm{HL} 7$ standards that simplifies the reporting workflow and enables near real time reporting in Korea. Method: We first analyze the current process of infectious disease reporting in Korea and identify its shortcomings in detail. Next we analyze KRFID (Korea Report Form of Infectious Disease) and related regulations to draw a data architecture design. Finally we take existing HL7 CDA implementation guides such as PHIN and Healthcare Associated Infection (HAI) Reports Template and conduct a comparative analysis to derive our design of CDA. Result: The final design of CDA consists of Patient and Infection sections. The Patient section includes 4 entries and the Patient section has 6 entries. KRFID is composed of 24 data items, of which 14 are included in the CDA header and the other in the body. The value of each entry is encoded using either SNOMED-CT or LOINC. Conclusion: The system we developed enables fast reporting by eliminating unnecessary workload and delays. In the reporting process, the steps for manual entry, printing a form, and sending it via FAX at healthcare providers can be omitted and the procedure in which employees at Regional Health Centers manually enter data through KCDC web portal can also be removed. The system also offers interoperability by using international standards. Specifically, we adopted HL7 CDA for the report form and LOINC and SNOMED-CT for encoding data. Finally, due to the regulatory requirement that all infectious disease reports should be documented and archived, the adoption of CDA as the electronic format of KRFID satisfies the regulation as well as the need for real time monitoring of infectious diseases in Korea.
\end{abstract}

\section{Keywords}

HL7, Surveillance, CDA, XDR, Interoperability

Correspondence to:

II-Kon Kim

Kyungpook National University Jung-gu, Deagu, Republic of Korea E-mail: ikkimgg@gmail.com

EJBI 2012; 8(4):12-15

\section{Introduction}

Due to advanced means of transportation and urbanization of societies, the spread of infectious diseases gets faster and faster nowadays and the importance of real time monitoring of such diseases becomes even bigger to prevent disastrous pandemic. At present, the regulatory process for reporting diagnosed infectious diseases in Korea [1] involves manual entry of data, paper-based reporting formats, obsolete delivery methods such as FAX, and a redundant two stage reporting (from healthcare providers to Regional Health Centers and back to KCDC (Korea Centers for Disease Control \& Prevention)).

Although a recent effort to modernize the process includes a web portal at KCDC allows that the reporting from Health Centers to KCDC can be done via internet, yet it requires manual entry of data, failing to use available electronic health records (EHRs). All these factors contribute to delayed reports and prevention of effective and real time monitoring of infectious diseases. 


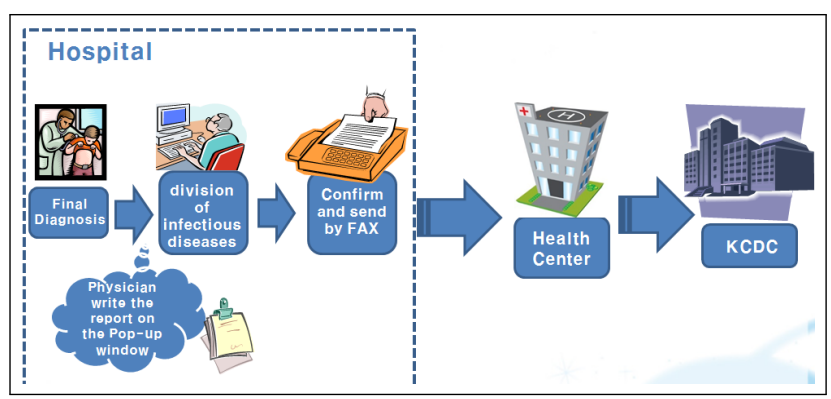

Figure 1: Workflow of infectious diseases report

Another problem with the current reporting process is that there is no regulatory requirement about the terminology and coding system for data included in the reports and it makes hard to process and analyze the data. The regulation also requires that all reports must be archived in the paper form at Regional Health Centers which should be taken into consideration in the design of new system.

In this paper, we design a new electronic infectious disease reporting system that addresses all the above issues with the current reporting process. The system offers a high level of interoperability by adopting international standards including HL7 CDA [2], SNOMED-CT (Systematized Nomenclature of Medicine - Clinical Terms) [3], and LOINC (Logical Observation Identifiers Names and Codes) 4. It is anticipated that the new system can help establishing a new regulatory process for infectious disease reporting and reduces the cost and time for endto-end reporting, leading to effective and near real time monitoring and surveillance of infectious diseases.

\begin{tabular}{|c|c|c|c|c|c|c|}
\hline \multicolumn{7}{|c|}{ Korea Report Form of Infectious Diseases } \\
\hline \multirow{4}{*}{$\begin{array}{c}\text { Personal } \\
\text { data }\end{array}$} & \multirow{2}{*}{\begin{tabular}{|l} 
Name \\
Phone \\
Number \\
\end{tabular}} & \multirow{2}{*}{\begin{tabular}{|l} 
Kil-dong Hong \\
0531111234 \\
\end{tabular}} & \multicolumn{2}{|c|}{$\begin{array}{l}\begin{array}{l}\text { Social Security } \\
\text { number }\end{array} \\
\end{array}$} & \multicolumn{2}{|c|}{$890101-123456 \mathrm{DE}$} \\
\hline & & & Occupation & Student & Gender & Male \\
\hline & Address & \multicolumn{5}{|l|}{ Deagu Korea } \\
\hline & \begin{tabular}{|l|} 
Unknown \\
address
\end{tabular} & $x$ & Unidentified & \multicolumn{3}{|l|}{$x$} \\
\hline $\begin{array}{l}\text { Name of } \\
\text { disease }\end{array}$ & \multicolumn{6}{|l|}{ Cholera } \\
\hline Onset date & \multicolumn{2}{|l|}{20111201} & $\begin{array}{l}\text { Diagnosis } \\
\text { date }\end{array}$ & \multicolumn{3}{|l|}{20120101} \\
\hline Test result & \multicolumn{6}{|c|}{ test not done } \\
\hline $\begin{array}{l}\text { Patient } \\
\text { Type }\end{array}$ & \multicolumn{2}{|l|}{ Confirmed } & \begin{tabular}{|l|} 
Inpatient \\
or \\
out patient
\end{tabular} & \multicolumn{3}{|l|}{ inpatient } \\
\hline \multirow{3}{*}{$\begin{array}{c}\text { Contact } \\
\text { with }\end{array}$} & \multirow{3}{*}{\multicolumn{2}{|c|}{ Infected group }} & \multirow{3}{*}{$\begin{array}{l}\text { Infection } \\
\text { location }\end{array}$} & Internal & \multicolumn{2}{|l|}{$x$} \\
\hline & & & & Abroad & Country & China \\
\hline & & & & & Period & 2 month \\
\hline $\begin{array}{l}\text { Death or } \\
\text { not }\end{array}$ & Alive & Y & Death reasor & & & \\
\hline \multirow{3}{*}{$\begin{array}{l}\text { Optional } \\
\text { record }\end{array}$} & Remarks & & & & & \\
\hline & $\begin{array}{l}\text { Facility } \\
\text { code }\end{array}$ & \multicolumn{5}{|l|}{123456} \\
\hline & $\begin{array}{l}\text { Doctor } \\
\text { name }\end{array}$ & Henry & $\begin{array}{l}\text { License } \\
\text { numbers }\end{array}$ & 123456 & & \\
\hline $\begin{array}{l}\text { Facility } \\
\text { name }\end{array}$ & \multicolumn{2}{|c|}{ Kyoung-pook hospital } & \begin{tabular}{|l|} 
Facility \\
director
\end{tabular} & \multicolumn{3}{|l|}{ Jhon } \\
\hline
\end{tabular}

Figure 2: CDA KRFID

\section{Methods}

We first analyze the current process of infectious disease reporting in Korea and identify its shortcomings in detail. The regulatory procedure for reporting in Korea is as follows. First, a physician upon detecting of an infectious disease reports to the QI (quality improvement) department. Second, the staff of the QI department reviews and confirms the report. Third, the QI department reports it to a Regional Health Center, using a fax, an e-mail or a paper format. Next, the Regional Health Center sends the report to the KCDC. The whole process is illustrated in Figure 1.

Next we analyze KRFID (Korea Report Form of Infectious Disease) [5] and related regulations to draw a data architecture design. There are 2 types of public health reporting (immediately and within 7days). And each type has a report form. In this paper, we are handle the immediately reporting type that requires near real time reporting.

Finally we take existing HL7 CDA implementation guides such as Healthcare Associated Infection (HAI) Reports Template [6] and Consolidated CDA Templates [7] and conduct a comparative analysis to derive our design of CDA. Table 1 lists the data items of KRFID and CDA location. The data items are coded using either LOINC that is a database and universal standard for identifying medical laboratory observations; or SNOMED-CT that is a systematically organized computer processable collection of medical terminology covering most areas of clinical information such as diseases, findings, procedures, microorganisms, pharmaceuticals etc.

Table 1: Lists the data items of KRFID and CDA location

\begin{tabular}{|c|c|}
\hline Data items & CDA location \\
\hline Name & \\
Parent name & \\
Phone number & \\
Gender & CDA head \\
Address & \\
Zip code & \\
Facility code & \\
Doctor name & \\
Facility name & \\
Director of Facility & \\
\hline Patient occupation & \\
Name of disease & \\
Onset date & \\
Diagnosis date & \\
Test result & CDA body \\
Patient type & \\
Contact with & \\
Infection location & \\
Reason of death & \\
Remarks & \\
\hline
\end{tabular}

\section{Results}

We started with the list of data items defined by the PHIN messaging guideline, which is compared with that of KRFID. We first noted that insurance-related data are 
not required by KRFID, which are, therefore, removed from out list.

Table 2: Data items coded using LOINC

\begin{tabular}{|c|c|}
\hline Data items & LOINC codes \\
\hline Name of disease & $29308-4 /$ Diagnosis \\
\hline Problem location & $56824-6 /$ Problem location \\
\hline Remarks & $51855-5 /$ NOTE \\
\hline
\end{tabular}

We next applied LOINC to code the newly added data items. Codes for only three data items (name of disease, problem location, and remarks) were found in LOINC, which and their corresponding LOINC codes are listed in Table 2. For those data items that could not be LOINCcoded, we applied SNOMED-CT. These data items include patient status, patient care status, test result and contact with. Table 3 lists the data items and their corresponding SNOMED-CT codes.

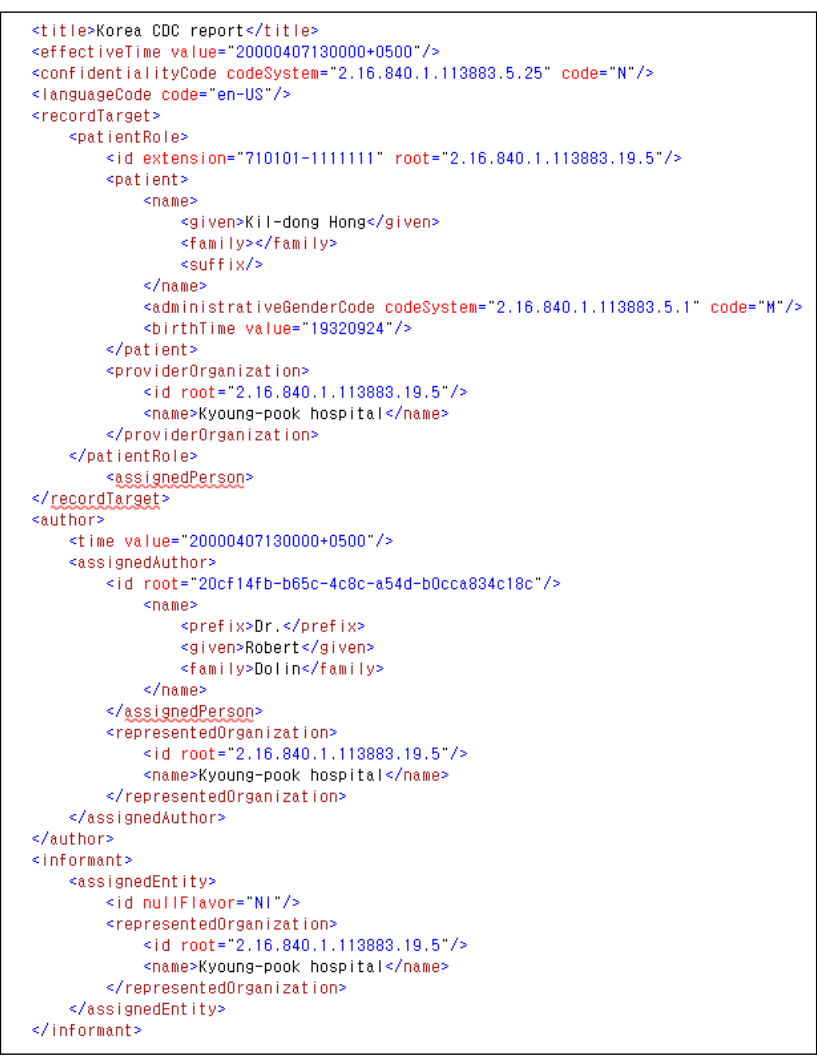

Figure 3: CDA Header

The HL7 CDA developed in this study contains two sections: infection and patient. The infection section consists of four entries and the patient section has six entries. KRFID has 24 data items, 14 of them are included in the CDA header and 10 of them included in the CDA body. Table 4 presents the sections and entries of the CDA we developed. The name of disease, test result and infection location commonly include onset date, diagnosis date and travel period as effectiveTime. Since the reporting system only considers information on patients and their diseases, the CDA entry type is fixed to be "observation". We applied SNOMED-CT to encode values.

Fig 2 illustrates a CDA example to report a case of a patient named Hong Kil-doing, a student at age 23, living in the city of Daegu, Korea, who visited Kyung-puk hospital on January 1st, 2012 and was diagnosed a cholera. Figure 3 is the CDA header and Figure 4 is the CDA body showing entries of diagnosis and laboratory test result only, omitting the rest for presentation purpose.

Table 3: Data items coded using SNOMED-CT

\begin{tabular}{|c|c|}
\hline Data items & SNOMED-CT codes \\
\hline Patient occupation & $184104002 /$ Patient occupation \\
\hline Status & $391741014 /$ Status \\
\hline Patient care status & $447503010 /$ Patient care status \\
\hline Test result & $2550471015 /$ Laboratory test result \\
\hline Contact with & $20251010 /$ Contact with \\
\hline
\end{tabular}

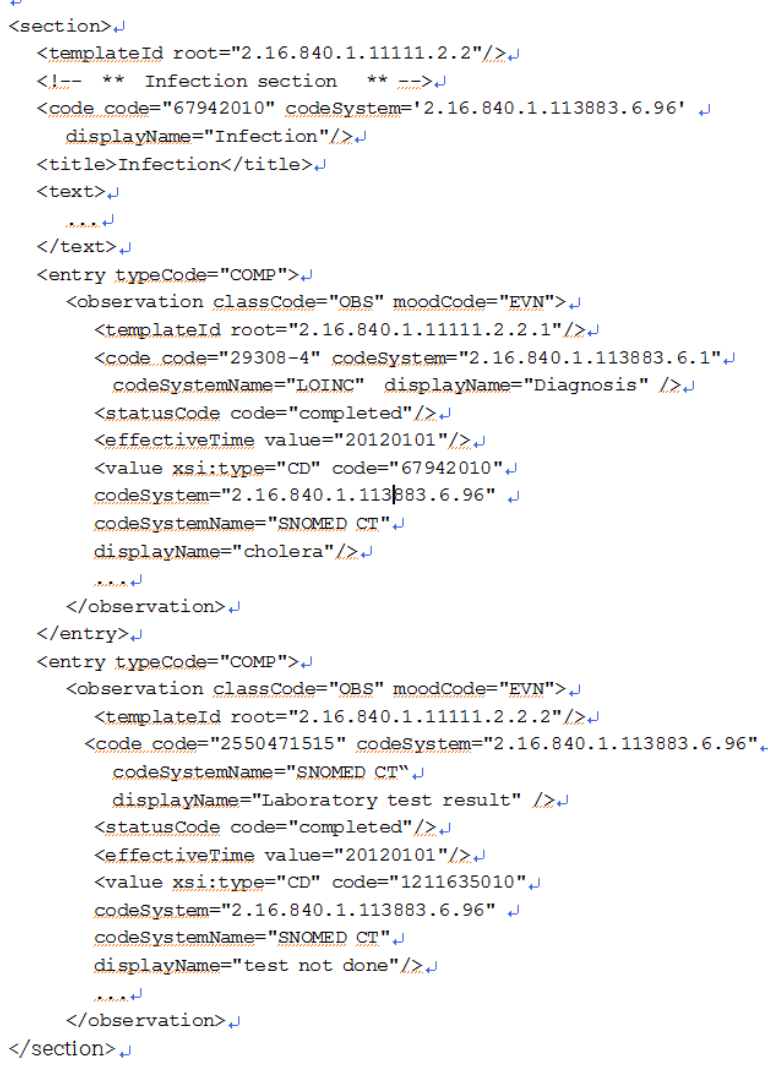

Figure 4: CDA Body

\section{Discussion}

The system we developed enables fast reporting by eliminating unnecessary workload and delays. In the reporting process, the steps for manual entry, printing a form, and sending it via FAX at healthcare providers can be omitted and the procedure in which employees at Regional Health Centers manually enter data through KCDC web portal can also be removed. Figure 5 presents the new workflow of infectious disease reporting based on CDA.

The system also offers interoperability by using international standards. Specifically, we adopted HL7 CDA for the report form and LOINC and SNOMED-CT for encoding data. Finally, due to the regulatory requirement that 
Table 4: CDA section and entry

\begin{tabular}{|c|c|c|}
\hline Section & Entry & Remarks \\
\hline \multirow{3}{*}{ Patient } & $\begin{array}{c}\text { Patient occupation } \\
\text { Patient status } \\
\text { Death observation } \\
\text { Reason of death }\end{array}$ & \\
\hline \multirow{3}{*}{ Infection } & Name of disease & Includes Onset date as effectiveTime \\
& Test result & Includes Diagnosis date as effectiveTime \\
& Patient type & \\
& Contact with & \\
& Infection location & Includes Travel priod as effectiveTime \\
& Remarks & \\
\hline
\end{tabular}

all infectious disease reports should be documented and archived, the adoption of HL7 CDA as the electronic format of KRFID satisfies the regulation as well as the need for real time monitoring of infectious diseases in Korea. [8]

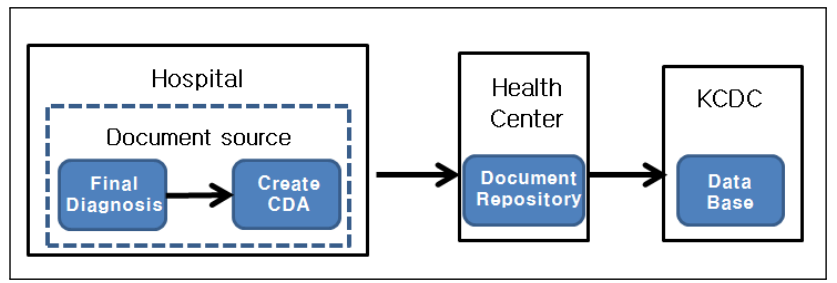

Figure 5: New workflow of infectious diseases report based on CDA

At present the application of the system is limited to the class of infectious diseases that are required to be reported immediately, but will cover the rest of diseases in the future. The current status of the project is final development stage and a pilot program to collect feedbacks and various usage statistics is being planned.

\section{Acknowledgements}

This work was supported by the IT R\&D program of MKE/KEIT. [10041145, Self-Organized Softwareplatform(SOS) for welfare devices]

\section{References}

[1] Korea Centers For Disease Control \& Prevention, http://www.cdc.go.kr, accessd June 2012

[2] HL7 International Version 3.0 Edition 2012

[3] Clinical Terminology Service, http://cliniclue.com accessed June 2012

[4] Logical Observation Identifiers Names and Codes (LOINC@), http://search.loinc.org accessed June 2012

[5] Case Definitions for National Notifiable Infectious Diseases 2011

[6] Implementation Guide for CDA Release 2.0 Consolidated CDA Templates (US Realm). December 2011

[7] HL7 Implementation Guide for CDA Release 2.0: Healthcare Associated Infection (HAI) Reports, Release 7 (US Realm). March 2012

[8] R. W. Pinner, "Public health surveillance and information technology". Emerg infect Dis. July-September 1998 